\title{
FAMILIES OF SU(2) REPRESENTATIONS FOR MAPPING CYLINDERS OF PERIODIC MONODROMY
}

\author{
by G. DASKALOPOULOS*, S. DOSTOGLOU AND R. WENTWORTH $\dagger$
}

(Received 21st September 1995)

\begin{abstract}
We examine the action of diffeomorphisms of an oriented surface with boundary on the space of conjugacy classes of $S U(2)$ representations of the fundamental group and prove that in the case of a single periodic diffeomorphism the induced action always has fixed points. For the corresponding 3-dimensional mapping cylinders we obtain families of representations parametrized by their value on the longitude of the torus boundary.
\end{abstract}

1991 Mathematics subject classification: 57N10, $14 \mathrm{H} 60$.

\section{Motivation and outline}

It is an open question whether for $K$ a knot in $S^{3}$ and $X$ its complement there always exist non-trivial representations of $\pi_{1}(X)$ into $S U(2)$.

Part of the importance of this question lies in its relevance to the following: Let $M_{n}$ be the homology 3-sphere obtained by $1 / n$-Dehn surgery on $K$. Then $K$ has property $\mathrm{P}$ if $\pi_{1}\left(M_{n}\right)$ is not trivial for any $n$. Bing and Martin conjectured 1971 that all non-trivial knots have property $P$.

The connection between property $\mathrm{P}$ and $S U(2)$ representations is as follows, cf. [5] and [6]: A representation $\rho: \pi_{1}(X) \rightarrow S U(2)$ restricts to give a representation of the fundamental group $\mathbb{Z} \oplus \mathbb{Z}$ of the torus boundary of $X$, described by $(\rho(m), \rho(l))$, the values of $\rho$ on the meridian $m$ and the longitude $l$ of the knot. Now the space $\mathcal{M}_{\partial}$ of all representations of the boundary, also known as character variety, is rather simple, a sphere with 4 distinguished points corresponding to the representations $( \pm 1, \pm 1)$, see figure.

According to the main theorem of [1], if $K$ fails to have property $\mathrm{P}$ this happens only for the \pm 1 surgeries. It is then enough to show that on $\mathcal{M}_{\partial}$ the curve of restricted representations of $X$ intersects the curves of the representations of the torus that extend to the \pm 1 surgeries of the knot.

Call a family $\rho_{\alpha}$ for $\alpha$ in $(0,1)$ of irreducible $S U(2)$ representations "good" if $\rho_{\alpha}(l)$ is conjugate to the element

* Supported in part by NSF Grant DMS-9504297

† Supported in part by NSF Grant DMS-9503635 and a Sloan Fellowship 


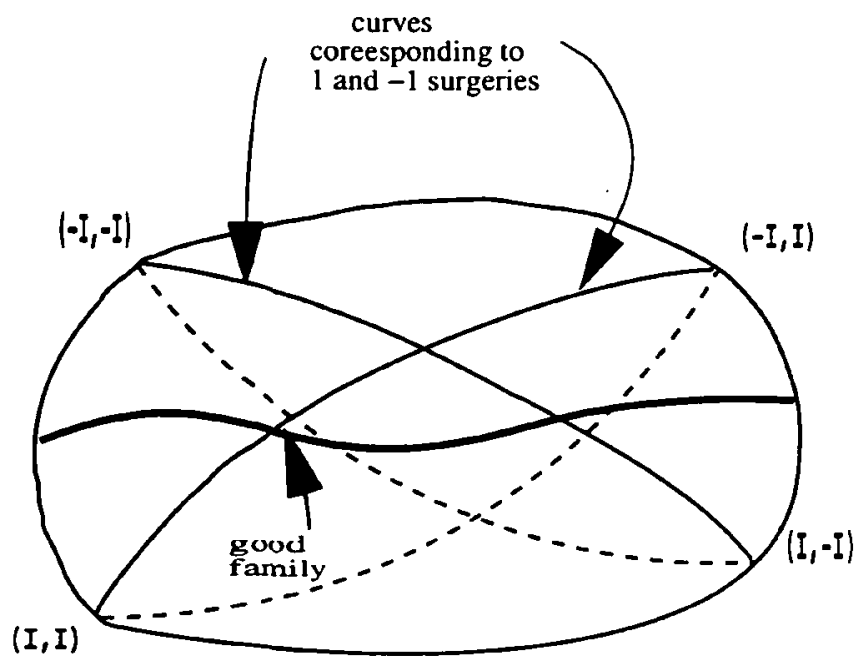

On the character variety of the boundary torus

$$
a=\exp \left(\begin{array}{cc}
\pi i \alpha & 0 \\
0 & -\pi i \alpha
\end{array}\right)
$$

In particular, good families are parametrized by their value on the longitude. Arguments in [6] (Propositions 1.4 and 1.6) show that on $\mathcal{M}_{\partial}$ a good family always intersects transversely the curves corresponding to \pm 1 surgeries, see figure. The existence of such a family for $\pi_{1}(X)$ would then imply property $\mathrm{P}$ for $K$. All the representation spaces of knots worked out so far contain good families.

Here we study three-manifolds $\Sigma_{h}$ with torus boundary, not necessarily knot complements, that are mapping cylinders of periodic monodromy $h$. This means that for an oriented surface $\Sigma_{0}$ with a single boundary component and $h$ a periodic automorphism of $\Sigma_{0}$ which is the identity on $l=\partial \Sigma_{0}$, we form

$$
\Sigma_{h}=\Sigma_{0} \times[0,1] /(z, 0) \sim(h(z), 1) .
$$

Let $\Re_{\alpha}$ be the space of conjugacy classes of representations of $\pi_{1}\left(\Sigma_{0}\right)$ into $S U(2)$ sending the boundary $l=\partial \Sigma_{0}$ to an element in $S U(2)$ conjugate to

$$
a=\exp \left(\begin{array}{cc}
\pi i \alpha & 0 \\
0 & -\pi i \alpha
\end{array}\right)
$$

cf. [2]. By the Seifert-van Kampen theorem, producing a good family for $\Sigma_{h}$ is equivalent to producing (in a continuous fashion) fixed points of the induced map 


$$
h_{\alpha}^{*}: \mathfrak{R}_{\alpha} \rightarrow \mathfrak{R}_{\alpha}
$$

on classes of representations of $\Sigma$, cf. [3]. The main result here is:

Theorem 1.1. If $h$ is periodic then $h_{\alpha}^{*}$ has fixed points for each $0 \leq \alpha \leq 1$.

The theorem is proved by identifying $\mathfrak{R}_{\alpha}$ with the moduli spaces of parabolic bundles and applying techniques of complex geometry (see section 2 and section 3 for details). It will be clear in the proof that periodicity of $h$ is needed exactly to make the identification with parabolic bundles.

The theorem and its proof yield

Corollary 1.2. If $h$ is periodic then for each $\alpha \in(0,1)$ there exists an irreducible $S U(2)$ representation of $\pi_{1}\left(\Sigma_{h}\right)$ which sends $(\partial \Sigma, *)$ to an element conjugate to a. Moreover, these representations fit into a good family.

Note that this is a statement for the mapping cylinder for any periodic monodromy, not just knot complements. Knots with periodic monodromy are the torus knots. For these knots, known to have property $P$, see [11] and [8], the theorem provides the new information that they, too, admit good families.

\section{Representation spaces and parabolic bundles}

Throughout the paper and unless otherwise mentioned $\Sigma$ will denote a compact oriented surface of genus $g \geq 2$.

Let $p$ be a point in $\Sigma$, and let $(\tilde{D}, z)$ be a local coordinate system at $p$, i.e.

$$
z: \tilde{D} \rightarrow D
$$

is a diffeomorphism with the unit disk $D \subset \mathbb{C}$. If we are considering a complex structure on $\Sigma$ we shall require $z$ to be complex analytic. Let $\Sigma_{0}=\Sigma \backslash\{p\}$. We shall use the same notation $\Sigma_{0}$ for the surface with boundary $\Sigma \backslash \tilde{D}$. Moreover, we set $\tilde{D}^{*}=\tilde{D} \backslash\{p\}$.

Let $E_{0} \rightarrow \Sigma_{0}$ denote a trivial complex rank 2 vector bundle with a Hermitian metric, and let $\mathcal{A}$ denote the space of smooth hermitian connections on $E_{0}$. We shall denote by $\mathcal{A}_{F} \subset \mathcal{A}$ the subspace of flat connections and by $\left(5=\operatorname{Map}\left(\Sigma_{0}, S U(2)\right)\right.$ the unitary gauge group of $E_{0}$ which acts on $\mathcal{A}$ by pull-back in the usual way. Note that $\mathfrak{G}$ preserves $\mathcal{A}_{\boldsymbol{F}}$. We topologize both $\mathcal{A}$ and $\mathbb{5}$ with the obvious Fréchet topologies. Let $\mathfrak{N}=\mathcal{A}_{F} / \mathfrak{G}$ with the quotient topology.

The holonomy map identifies $\mathfrak{N}$ with the space $\Re$ of conjugacy classes of representations of $\pi_{1}\left(\Sigma_{0}\right)$ to $S U(2)$. Given a real number $0 \leq \alpha \leq 1$, let $\mathfrak{N}_{x} \subset \mathfrak{N}$ denote the subspace of equivalence classes of connections on $E_{0}$ with holonomy $a=\exp (\pi i \hat{\alpha})$ where $\hat{\alpha}$ is the matrix $\operatorname{diag}(\alpha,-\alpha)$. Clearly, the holonomy homomorphism identifies $\mathfrak{N}_{x}$ 
with $\Re_{x}$, the subspace of $\Re$ consisting of representations sending a loop $\lambda$ around $p$ to an element in $S U(2)$ conjugate to $a$.

From now on we will identify $\mathfrak{N}$ with $\mathfrak{R}$ and $\mathfrak{N}_{\alpha}$ with $\mathfrak{R}_{x}$. It follows that for $\alpha \neq 0,1, \mathfrak{N}_{\alpha} \simeq \mathfrak{R}_{x}$ is a smooth manifold of real dimension $6 g-4$. Recall that for $\alpha=1, \Re_{\alpha}$ is also smooth but of dimension $6 g-6$, whereas for $\alpha=0, \Re_{\alpha}$ is a real analytic variety of dimension $6 g-6$ which is smooth only for $g=2$ (cf. [12]).

Slightly less immediate is the task of putting a complex structure on $\mathfrak{R}_{x}$. This can be done via the theorems of Narasimhan-Seshadri and Mehta-Seshadri which we will briefly review (see [12] and [9] for more details). Suppose now that $\Sigma$ is a Riemann surface. Recall that a holomorphic structure on a vector bundle $E \rightarrow \Sigma$ is equivalent to a $\bar{\partial}$-operator acting on smooth sections: $\bar{\partial}_{E}: \Omega^{0}(E) \rightarrow \Omega^{0,1}(E)$. Then the following is standard.

Definition 2.2. Let $\left(E, \bar{\partial}_{E}\right)$ be a holomorphic rank 2 vector bundle on $\Sigma$ (we will usually omit the notation $\bar{\partial}_{E}$ when the holomorphic structure is understood). Then $E$ is called stable (resp. semistable) if for any line subbundle $L \subset E$ we have

$$
\operatorname{deg} L<\frac{1}{2} \operatorname{deg} E \quad(\text { resp. } \leq)
$$

Let $\mathfrak{M}_{0}$ and $\mathfrak{M}_{1}$ denote the spaces of equivalence classes of semistable vector bundles on $\Sigma$ with determinants isomorphic to $\mathcal{O}$ and $\mathcal{O}(-p)$, respectively, where $\mathcal{O}$ denotes the trivial holomorphic line bundle, and $\mathcal{O}(-p)$ the line bundle with divisor $\{-p\}$. Narasimhan and Seshadri prove that $\mathfrak{M}_{0}$ and $\mathfrak{M}_{1}$ are projective varieties homeomorphic to $\mathfrak{R}_{0}$ and $\mathfrak{R}_{1}$, respectively. The isomorphisms $\mathfrak{R}_{0} \simeq \mathfrak{M}_{0}$ and $\mathfrak{R}_{1} \simeq \mathfrak{M}_{1}$ are given by sending a given unitary connection to the associated $\bar{\partial}$-operator. Note that since we are working on a Riemann surface there is no integrability condition.

In the case of $\mathfrak{M}_{1}$ more can be said (cf. [12]). Indeed, $\mathfrak{M}_{1}$ is a fine moduli space, i.e. it admits a universal bundle. More precisely, there is a holomorphic bundle $U \rightarrow \Sigma \times \mathfrak{M}_{1}$ with the property that the restriction of $U$ to $\Sigma \times\{E\}$ is isomorphic to $E$. Let $\pi_{1}: \mathfrak{S} \rightarrow \mathfrak{M}_{1}$ denote the restriction of the projective bundle $\mathbb{P}(U)$ to $\{p\} \times \mathfrak{M}_{1}$. The next result is due to Mehta-Seshadri ([9, Theorem 5.3]).

Proposition 2.3. For any $\alpha \neq 0,1, \mathfrak{R}_{\alpha}$ is diffeomorphic to $\mathfrak{H}$. Hence, $\mathfrak{R}_{\alpha}$ admits the structure of a smooth projective variety. Moreover, there is a morphism $\pi_{0}: \mathfrak{G} \rightarrow \mathfrak{M}_{0}$ which is a $\mathbb{P}^{1}$ bundle when restricted the stable points of $\mathfrak{M}_{0}$.

The diagram

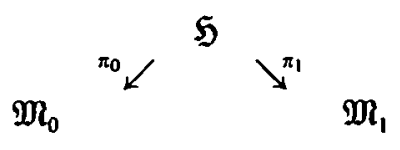

is called the Hecke correspondence [10]. Since our main result relies heavily on it, we 
will briefly explain this construction from two different points of view.

First, from the algebraic point of view observe that given an equivalence class $E \in \mathfrak{N}_{1}$, the fibre of $\pi_{1}$ corresponds to the exact sequence

$$
E^{*} \stackrel{\iota}{\longrightarrow} \mathbb{C}_{p} \longrightarrow 0
$$

where $\mathbb{C}_{p}$ denotes the torsion sheaf on $\Sigma$ with rank 1 support at $p$. Therefore, $\mathfrak{5}$ can be regarded as the set of equivalence classes of pairs $(E, \ell)$, where $E$ is a stable bundle of degree -1 and $\ell$ is a homomorphism as in (2.4). Moreover, $\pi_{1}(E, \ell)=E$. Pairs $(E, \ell)$ as above are called parabolic bundles, and $\mathfrak{S}$ is called the moduli space of parabolic bundles.

Lemma 2.5. Given $(E, \ell) \in \mathfrak{H}$, let $F^{*}$ denote the kernel of $\ell$ in (2.4). Then $F$ is semistable with trivial determinant.

Proof 2.5. Since $\Lambda^{2}(E) \simeq \mathcal{O}(-p)$, it follows that $\Lambda^{2}(F) \simeq \mathcal{O}$. Any subbundle $L \subset F^{*}$ is also a subbundle of $E^{*}$, and hence

$$
\operatorname{deg} L \leq \operatorname{deg} E^{*} / 2=1 / 2 \Longrightarrow \operatorname{deg} L \leq 0,
$$

from which we conclude $F^{*}$, and therefore also $F$, is semistable.

The map $\pi_{0}$ is defined by $\pi_{0}(F, \ell)=\operatorname{gr}(F)$, where $\operatorname{gr}(F)$ is the usual graded bundle associated to a Seshadri filtration of the semistable bundle $F$ (cf. [12]). If $F$ is stable then the fibre of $\pi_{0}$ over $F$ is easy to compute: from the sequence

$$
0 \longrightarrow F^{*} \longrightarrow E^{*} \stackrel{\iota}{\longrightarrow} \mathbb{C}_{p} \longrightarrow 0
$$

we see that $\pi_{0}^{-1}(F)$ is isomorphic to

$$
\mathbb{P}\left(\operatorname{Ext}^{1}\left(\mathbb{C}_{p}, F^{*}\right)\right) \simeq \mathbb{P}\left(F_{p}^{*}\right) \simeq \mathbb{P}^{1},
$$

i.e. $\pi_{0}$ is $\mathbb{P}^{1}$ fibration over the stable part of $\mathfrak{M}_{0}$. We now describe the fibre of $\pi_{0}$ over the most singular point of $\mathfrak{M}_{0}$.

Proposition 2.6. Let $f_{0}$ denote the class of the trivial bundle $\mathcal{O} \oplus \mathcal{O}$ in $\mathfrak{M}_{0}$. Then $\pi_{0}^{-1}\left(f_{0}\right) \simeq \mathbb{P}^{g-1}$.

Proof. Consider extensions

$$
\begin{aligned}
& 0 \longrightarrow \mathcal{O} \rightarrow F \rightarrow \mathcal{O} \rightarrow 0 \\
& 0 \longrightarrow E \rightarrow F \rightarrow \mathbb{C}_{p} \rightarrow 0 .
\end{aligned}
$$


We check under what circumstances $E$ is stable. First, if (2.7) is the trivial extension then an appropriate change of basis will give a non-zero map $0 \rightarrow \mathcal{O} \rightarrow E$ whose image would then destabilize $E$. Hence, we suppose the extension (2.7) is non-trivial. In terms of $\bar{\partial}$-operators, we write

$$
\bar{\partial}_{F}=\left(\begin{array}{ll}
\bar{\partial} & \beta \\
0 & \bar{\partial}
\end{array}\right), \quad \beta \in H^{0,1}(\Sigma), \beta \neq 0 .
$$

The extension (2.8) is determined by a choice of line $\ell=\left(\begin{array}{l}\xi \\ \eta\end{array}\right) \in F_{p}^{*} \simeq \mathbb{C} \oplus \mathbb{C}$. If $\xi=0$, then the holomorphic section $\phi=\left(\begin{array}{l}1 \\ 0\end{array}\right)$ of $F$ lifts to a non-zero map $0 \rightarrow \mathcal{O} \rightarrow E$, and $E$ is unstable as before. Suppose $\ell=\left(\begin{array}{l}1 \\ 0\end{array}\right)$. We first show that $E$ is stable with this choice, and then we show that up to automorphism this choice is unique. By (2.7), $F$ is clearly semistable of degree zero. If $L \subset E$ is a line subbundle of non-negative degree, then its image in $F$ would either have positive degree, contradicting semistability, or define a holomorphic section of $F$ independent of $\phi$, of which there aren't any. Thus $E$ is stable. Now suppose that $\ell=\left(\begin{array}{l}\xi \\ \eta\end{array}\right)$ with $\xi \neq 0$. The gauge transformation

$$
\left(\begin{array}{ll}
1 & 0 \\
\lambda & 1
\end{array}\right)
$$

preserves the extension (2.9) and takes $\ell$ to $\left(\begin{array}{c}\xi \\ \lambda \xi+\eta\end{array}\right)$. Hence, for an appropriate choice of $\lambda$ we may eliminate $\eta$. Then by an action of the diagonal matrices $\simeq \mathbb{C}^{*}$ which also preserves the extension, we may assume $\xi=1$. Therefore, the choice of flag $\ell$ is unique. Finally, the gauge transformation $\operatorname{diag}\left(\lambda, \lambda^{-1}\right)$ for $\lambda \in \mathbb{C}^{*}$ acts on the extensions (2.9) by $\lambda^{2}$. The equivalence classes of stable degree 1 bundles obtained in this way (by taking $\left.E^{*}\right)$ are thus in one-to-one correspondence with $\mathbb{P}\left(H^{0,1}(\Sigma)\right) \simeq \mathbb{P}^{g-1}$.

We mentioned before that the Narasimhan-Seshadri isomorphisms

$$
\begin{aligned}
& \psi_{0}: \mathfrak{R}_{0} \longrightarrow \mathfrak{M}_{0} \\
& \psi_{1}: \mathfrak{R}_{1} \longrightarrow \mathfrak{M}_{1},
\end{aligned}
$$

are given by $\psi_{i}[\nabla]=\left[\bar{\partial}_{\nabla}\right]$, where $\bar{\partial}_{\nabla}$ is the $\bar{\partial}$-operator associated to the connection $\nabla$. We would like now to briefly describe the Mehta-Seshadri isomorphism

$$
\psi_{\alpha}: \mathfrak{R}_{\alpha} \rightarrow \mathfrak{S}
$$

First, choose a unitary frame for $E$ over $\tilde{D}^{*}$ and define a continuous family $\left\{g_{\alpha}: \alpha \in(0,1)\right\}$ of complex gauge transformations by

$$
g_{\alpha}(z)= \begin{cases}I & \text { if } z \in \Sigma \backslash \tilde{D} \\ \operatorname{diag}\left(|z|^{\alpha / 2},|z|^{1-\alpha / 2}\right) & \text { if } z \text { near } p\end{cases}
$$


For $[\nabla] \in \Re_{\alpha}$, choose a representative $\nabla \in \mathcal{A}_{F}$, the flat connections on $\Sigma \backslash\{p\}$, such that on $\tilde{D}^{*}, \nabla=d+i(\hat{\alpha} / 2) d \theta$. Let $\bar{\partial}_{\nabla}$ be the $\bar{\partial}$-operator associated to $\nabla$. It is easy to see that $g_{\alpha}\left(\bar{\partial}_{\nabla}\right)$ extends to a holomorphic structure on a vector bundle $E$ of degree -1 . Set $\psi_{x}[\nabla]=\left[g_{a}\left(\bar{\partial}_{\nabla}, \ell\right)\right]$, where $\ell$ is the flag determined by the choice of frame. Moreover, it is easy to check that under the isomorphism above, $\pi_{1} \psi_{\alpha}[\nabla]=\left[g_{\alpha}\left(\bar{\partial}_{\nabla}\right)\right]$. The map $\pi_{0}$ can be viewed in a similar way: we set $\pi_{0}[\nabla]=\left[h_{x}\left(\bar{\partial}_{\nabla}\right)\right]$, where

$$
h_{\alpha}(z)= \begin{cases}I & \text { if } z \in \Sigma \backslash \tilde{D} \\ \operatorname{diag}\left(|z|^{\alpha / 2},|z|^{-\alpha / 2}\right) & \text { if } z \text { near } p .\end{cases}
$$

The identification $\psi_{\alpha}$ will play an important role in the next section.

\section{Invariant representations}

Using the results of the previous section we prove

Theorem 3.1. Let $\Sigma$ be a compact Riemann surface of genus $g \geq 2$ and $h: \Sigma \rightarrow \Sigma a$ complex automorphism of $\Sigma$ preserving a base point $\{p\}$. Let $H: \mathfrak{S} \rightarrow \mathfrak{G}$ denote the induced map $H[F, \ell]=\left[h^{*} F, \ell\right]$. Then $H$ has a fixed point.

Proof. Let $f_{0}$ be the class of the trivial bundle as in (2.6). Clearly, $f_{0}$ is a fixed point of the induced map $H_{0}: \mathfrak{M}_{0} \rightarrow \mathfrak{M}_{0}$ defined by $H_{0}[E]=\left[h^{*}(E)\right]$. On the other hand the following diagram commutes:

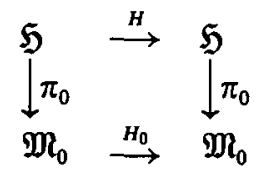

Indeed, let $[E, \ell] \in \mathfrak{S}$ with $\pi_{0}(E)=F$. This means that we have an exact sequence

$$
0 \rightarrow F^{*} \longrightarrow E^{*} \stackrel{\iota}{\longrightarrow} \mathbb{C}_{p} \longrightarrow 0 .
$$

We must show that the kernel $\tilde{F}^{*}$ of the map $h^{*}\left(E^{*}\right) \stackrel{\iota}{\longrightarrow} \mathbb{C}_{p} \longrightarrow 0$ is $h^{*}\left(F^{*}\right)$. Away from $p$, we clearly have $\tilde{F}^{*}=h^{*}\left(F^{*}\right)$. On the other hand, since $h^{*}$ is identity at $p$, the same is true at $p$. Hence, (3.2) commutes.

As a consequence, by Proposition 2.6, $H$ induces a holomorphic map

$$
H: \mathbb{P}^{g-1} \simeq \pi_{0}^{-1}\left(f_{0}\right) \longrightarrow \pi_{0}^{-1}\left(f_{0}\right) \simeq \mathbb{P}^{g-1} .
$$

Now such a map always has a fixed point, see Theorem 4 of [4].

If $h: \Sigma \rightarrow \Sigma$ is periodic, by Nielsen realization (see [7]) we may choose a complex 
structure on $\Sigma$ with respect to which $h$ is a complex automorphism. For the MehtaSeshadri isomorphism defined using this complex structure, the previous theorem applies with the following corollary:

Corollary 3.3 Let $\Sigma$ be a compact oriented surface of genus $g \geq 2$ and $h: \Sigma \rightarrow \Sigma a$ periodic diffeomorphism of $\Sigma$ preserving a base point p. Then the induced map $h_{\alpha}^{*}: \mathfrak{R}_{\alpha} \rightarrow \mathfrak{R}_{\alpha}$ has a fixed point for $0<\alpha<1$.

Proof. Since the maps $h_{\alpha}^{*}$ are invariant under isotopies, we may assume without loss of generality that $h$ is the identity in a neighbourhood of $p$ containing $\tilde{D}$. Let $\psi_{\alpha}: \mathfrak{R}_{\alpha} \rightarrow \mathfrak{H}$ denote the Mehta-Seshadri isomorphism with respect to the complex structure that renders $h$ a complex automorphism. We claim that the following diagram commutes:

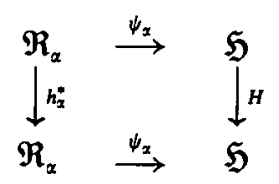

Indeed, $H \psi_{\alpha}[\nabla]=H\left[g_{\alpha} \nabla^{0,1}, \ell\right]=\left[h^{*} g_{\alpha} \nabla^{0,1}, \ell\right]=\left[g_{\alpha} h^{*} \nabla^{0,1}, \ell\right]=\psi_{\alpha} h^{*}[\nabla]$, where $g_{\alpha}$ is the gauge transformation defined in (2.10) and the third equality holds because of the special choice of $h$ at the beginning of the proof. The existence of a fixed point of $h_{\alpha}^{*}$ now follows from Theorem 3.1 and commutativity of the diagram (3.4).

Now let $\Re=\operatorname{Hom}\left(\pi_{1}(\Sigma), S U(2)\right) / S U(2)$ as before, and let $q: \Re \rightarrow[0,1]$ be the map $q(\rho)=\rho(\mu)$, where $\mu$ denotes the homotopy class of a loop around $p$. Let $\mathfrak{R}^{\prime}=\mathfrak{R} \backslash q^{-1}\{0,1\}$, and let

$$
\psi: \mathfrak{R}^{\prime} \longrightarrow(0,1) \times \mathfrak{H}
$$

denote the map $\psi(\rho)=\left(q(\rho), \psi_{q(\nabla)}(\rho)\right)$. In other words, note that

$$
\mathfrak{R}^{\prime}=\bigcup_{\alpha \in(0,1)} \mathfrak{R}_{\alpha}, \quad \psi=\bigcup_{\alpha \in(0,1)} \psi_{\alpha} .
$$

Let $h^{*}: \Re^{\prime} \rightarrow \mathfrak{R}^{\prime}$ denote the induced map $h^{*}=\cup_{\alpha \in(0,1)} h_{\alpha}$. The commutativity of (3.4) yields the commutativity of

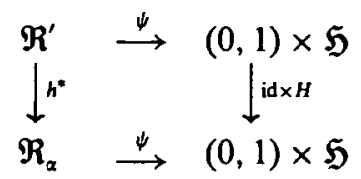


Hence, Theorem 3.1 immediately implies

Corollary 3.6. Let $h$ be as in Corollary 3.3. There is a continuous family $\left\{\rho_{\alpha}: \alpha \in(0,1)\right\}$ of fixed points of $h^{*}$ in $\Re^{\prime}$.

Remark 3.7. The family of Corollary 3.6 actually extends continuously over $\alpha=1$. Indeed, the inverse $\psi^{-1}:(0,1) \times \mathfrak{H} \rightarrow \mathfrak{R}^{\prime} \subset \mathfrak{R}$ of (3.5) extends continuously over $\alpha=1$ by setting $\psi^{-1}\left(\alpha,\left(\bar{\partial}_{E}, \ell\right)\right)=g_{1}\left(\bar{\partial}_{E}\right)$, where $g_{1}$ is defined as in (2.10) with respect to the flag $\ell$. Moreover, the commutativity of the diagram

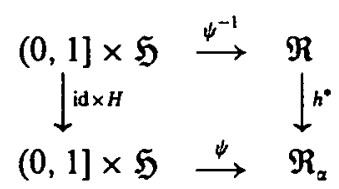

implies the remark.

\section{The 3-dimensional application}

As an application of these results, consider the question of the existence of $S U(2)$ representations of 3-manifolds.

Consider the mapping cylinder $\Sigma_{0} \times[0,1] /(z, 0) \times(h(z), 1)$, where $\Sigma_{0}=\Sigma \backslash\{p\}$ is an oriented surface of genus $g$ with $\partial \Sigma_{0}=K$ and $h: \Sigma \rightarrow \Sigma$ is an orientation preserving diffeomorphism with $h(p)=p$.

Let $\left\{a_{i}, b_{i}: i=1, \ldots, g\right\}$ be a set of generators for $\pi_{1}\left(\Sigma_{0}\right)$ which form a symplectic basis in $H_{1}\left(\Sigma_{0}, \mathbb{R}\right)$. It follows by a direct application of the Seifert-Van Kampen theorem that

$$
\pi_{1}(X)=\left\{a_{i}, b_{i}, t: h_{*}\left(a_{i}\right)=t a_{i} t^{-1}, h_{*}\left(b_{i}\right)=t b_{i} t^{-1}, i=1, \ldots, g\right\} .
$$

Therefore conjugacy classes of representations of $\pi_{1}(X)$ satisfy $\left[h_{*}(r)\right]=[r]$, i.e. they are fixed points of $h_{*}$.

Corollary 4.2. Let $K \subset M$ be a mapping cylinder with periodic monodromy. Then the fundamental group of the complement of $K$ in $M$ admits a good family of irreducible $S U(2)$ representations.

Proof. The only point to justify is the irreducibility. However, as representations of the surface they are irreducible, since they have non-trivial holonomy around a bounding cycle. Therefore, the corresponding representations of the complement are irreducible as well. 


\section{Concluding remarks}

The next step is to show that similar results hold for any $h$ and in so doing prove property $\mathbf{P}$ for all fibred knots, i.e. knots with mapping cylinder boundary.

It is clear from the proof of Corollary 3.3 that periodicity brought us to the holomorphic setting where we had use of the holomorphic maps $\mathfrak{R}_{z} \rightarrow \mathfrak{R}_{0}$ and $\mathfrak{R}_{\alpha} \rightarrow \mathfrak{R}_{1}$. To work without the periodicity assumption, it would be useful to have a direct description of these maps in terms of representations spaces.

\section{REFERENCES}

1. M. Culler, C. McA. Gordon, J. Luecke, and P. Shalen, Dehn surgery on knots, Ann. of Math. 125, (1987), 237-300.

2. G. Daskalopoulos and R. Wentworth, Geometric quantization for the moduli space of vector bundles with parabolic structure, unpublished.

3. S. Dostoglou and D. SAlamon, Instanton homology and symplectic fixed points, (London Math. Soc. Lecture Notes 192, 1994), 57-93.

4. T. Frankel, Manifolds with positive curvature, Pacific J. Math. 11 (1961), 165-174.

5. C. Frohman, Unitary representations of knot groups, Topology 32 (1993), 121-144.

6. C. Frohman and D. LoNG, Casson's invariant and surgery on knots, Proc. Edinburgh Math. Soc. 35 (1992), 383-395.

7. S. KeRCKHOFF, The Nielsen realization problem, Ann. of Math. 117 (1983), 235-265.

8. L. MoSer, Elementary surgery along a torus knot, Pacific J. Math. 38 (1971), 737-745.

9. V. Mehta and C. Seshadri, Moduli of vector bundles on curves with parabolic structures, Math. Ann. 248 (1980), 205-239.

10. M. S. Narasimhan and S. Ramanan, Geometry of Hecke cycles - I, in C. P. Ramanujam: A Tribute, (Springer-Verlag, New York, 1978).

11. H. SeIfert, Uber das Geschlecht von Knoten, Math. Ann. 110 (1934), 571-592.

12. C. Seshadri, Fibrés vectoriels sur les courbes algébriques, Astérisque 96 (1982).

Department of Mathematics

BROWN UNIVERSITY

PROVIDENCE

RI 02912

U.S.A.
Department of Mathematics

UNIVERSITY OF MISSOURI

Columbia

MO 65211

U.S.A.

Department of Mathematics

UNIVERSITY OF CALIFORNIA AT IRVINE

IR VINE

CA 92717

U.S.A. 